\title{
Körpervolum- und Körperdichtebestimmung am lebenden Säugling. \\ Zu ihrer Bedeutung und Methodik.
}

\author{
Von \\ M. Pfaundler. \\ (Aus der Universitäts-Kinderklinik in München.) \\ Mit 1 Textfigur und 1 Tafel. \\ (Eingegangen am 23. November 1911.)
}

Die Gesichtspunkte, die den von Kastner in vorstehender Arbeit mitgeteilten Bestimmungen zugrunde lagen, sind im wesentlichen folgende. Zunächst schien es von physiologischem Interesse, Normalzahlen für das Volumen und das spezifische Gewicht von gesunden Neugeborenen und Säuglingen festzulegen, da solche, als die Untersuchungen begannen, noch so gut wie gar nicht erhoben waren. Ferner dachten wir daran, daß das spezifische Gewicht möglicherweise einen ziffernmäßigen Hinweis auf Besonderheiten des Gesamtkörperbestandes in materieller Hinsicht liefern könnte. Man weiß, daß sich der Körper aus einzelnen Teilen, nämlich verschiedenen Gewebssystemen, Organen, Ballaststoffen, Säften usw., zusammensetzt, die in ihrem spezifischen Gewicht zum Teil beträchtlich voneinander abweichen. So beträgt z. B. das spezifische Gewicht der Knochenrindensubstanz fast 2,0, jenes der quergestreiften Muskulatur etwa 1,04, jenes des Fettgewebes etwa 0,97 (bezogen auf Wasser bei $4^{\circ} \mathrm{C}=1$ ). Es ist klar, daß Verschiebungen in dem wechselseitigen Mengenverhältnisse dieser einzelnen Teile auch in dem spezifischen Gewicht des Gesamtkörpers zum Ausdruck kommen müssen. Für die Frage jener konstitutionellen und sonstigen Anomalien in dem wechselseitigen Mengenverhältnis der Körperkonstituenten, die Schlo $B$ neuerdings als Korrelationsstörungen bezeichnet, war also einiger Aufschluß zu erhoffen. Endlich versprachen wir uns insbesondere von fortlaufenden Bestimmungen an einem und demselben Kinde bemerkenswerte Ergebnisse. Wir dachten, daß die 
Ergänzung der allenthalben üblich gewordenen Körpergewichtskurve durch eine Körpervolumkurve und die daraus sich ergebenden fortlaufenden Veränderungen der Körperdichte einen bedeutsamen Kommentar zu Schwankungen des absoluten Gewichtes bringen würden. Man weiß, daß z. B. Körpergewichtsstürze bei Säuglingen oft sehr Yerschiedenes bedeuten und klinisch eine sehr verschiedene Dignität haben. Es kann sich handeln um eine Exsiccation, um den Rückgang eines labilen Ansatzes, eine echte Körpereinschmelzung usw. Theoretisch müssen diese verschiedenen Typen durch verschiedenes Verhalten der Volumkurve bei gleichem Gewichtskurven. verlauf unterscheidbar sein, weil die zu Verlust gehenden Massen auf gleiches Gewicht verschiedenes Volum haben.

Die Ergebnisse von Kastners sorgfältigen Bestimmungen waren schon hinsichtlich der beiden erstgenannten Punkte leider wenig befriedigend. Bei der Durchsicht der nach aufsteigender Dichte geordneten Generaltabelle bemerkt man, daß wider Erwarten die als physiologisch erachteten Fälle fast über die ganze Tabelle zerstreut sind; sie finden sich schon in ihrem ersten Sechstel, und noch der allerletzte, der extrem hohe Wert, gehört ihnen zu. Wie Kastuer bemerkt, hat die Berechnung von Mittelzahlen unter diesen Umständen höchstens beschränkten Wert - jedenfalls lassen sich richtige "Standardzahlen“" nicht gewinnen. Dies wird auch nicht viel anders, wenn man die hier eingerechneten Fälle so scharf unter die klinische Lupe nimmt, wie dies neuerdings verlangt wird, und es uns eine lange fortgesetzte Beobachtung auch oft ermöglichte, oder wenn man nach Alter, Ernährungsmodus usw. sichtet.

Fast ebenso regellos zerstreut trifft man in jener Liste pathologische Fälle, die klinisch zweifellos zusammengehören, also etwa die pastösen, die lymphatischen, die exsudativen, die rachitischen, die intoxizierten, die ,dekomponierten“ Individuen. So zählt beispielsweise

zu den Atrophikern

,. .. Rachitikern

, , Pastösen und

,. ., (infektiös) Intoxizierten sowohl der Fall $16(\mathrm{D}=0,9115)$,

$$
\text { als ", , } 148(\mathrm{D}=1,0682) \text {, }
$$$$
\text { sowohl ", , } 22(\mathrm{D}=0,9180) \text {, }
$$$$
\text { als , , } 146(\mathrm{D}=1,0680) \text {, }
$$$$
\text { sowohl " " } 12(\mathrm{D}=0,9086) \text {, }
$$$$
\text { als , , } 147(\mathrm{D}=1,0680) \text {, }
$$

sowohl ," , $8(\mathrm{D}=0,9043)$,

als ", , $114(\mathrm{D}=0,9862)$. 
Fon der erwarteten Gruppenbildung und Sonderung m. e. W. ron einer durchgreifenden Gesetzmäßigkeit tritt auch hier nichts zutage, und die eingehende zahlenmäßige Bearbeitung ergibt nur wenig brauchbare Anhaltspunkte. Von welchem Gesichtspunkte aus immer man die Ergebnisse betrachte, allenthalben stößt man auf Unebenmäßigkeiten, ja scheinbar freie Willkür.

Wir mußten so zum Schlusse kommen, daß neben den Faktoren der Konstitution (Korrelation), des Alters, der Erkrankung im Körper noch ein weiterer Faktor auf das spezifische Gewicht Einfluß nehme, und zwar in bestimmender Weise. Diesen zu eruieren und, wenn möglich, auszuschalten, war also unsere Aufgabe. Verschiedene Wege führten uns auf die Spur; zunächst die folgende t'berlegung.

Nach den vorliegenden Ermittlungen (s. Vierordts Tabellen) haben die sämtlichen Organe und Gewebssysteme des Körpers, sowie auch die Gewebssäfte eine größere Dichte als der Gesamtkörper (Durchschnittswerte unserer Untersuchungen); nur jene des Fettgewebes kommt der letzteren ziemlich gleich. Es muß also bei der Bestimmung der Dichte des Gesamtkörpers etwas spezifisch Leichteres mit im Spiele sein. Dies ist offenbar das in verschiedenen Körperhöhlen eingeschlossene Gasgemenge (Luft und anderes). Bei gänzlicher Luftleere der Lungen und des Darmkanals bestimmte Kra use die Körperdichte erwachsener Leichen in der Tat auf den sehr hohen Wert von 1,1291. Daß nun im Gasgehalte und seinen zufälligen Veränderungen auch der alle Gesetzmäßigkeit störende Faktor gelegen sei, wurde schon dadurch sehr wahrscheinlich, daß ein bestimmender Einfluß auf die Gesamtdichte naturgemäß am ehesten von einer Substanz zu gewärtigen ist, deren Dichte von jener der übrigen Körperbestandteile sehr stark abweicht, einer anderen Größenordnung angehört.

Für den entscheidenden Einfluß gasförmiger Körperfüllsel auf die Gesamtkörperdichte sprechen ferner die bei einem und demselben Individuum binnen kurzer Zeiträume angetroffenen Differenzen. So fand z. B. Kastner das spezifische Gewicht im Falle Ehrenreich am 109. Lebenstage 0,8972 gegen 0,9697 am 102. Lebenstage (Differenz $0,0725)$, im Fall Ries am 56. Lebenstage 1,011 gegen 0,970 am 64. Tage (Differenz 0,041), im Falle Braun am 50. Lebenstage 0,9847 gegen 0,9322 am 56. Tage (Differenz 0,0525), wobei aber weder das klinische Verhalten der Individuen, noch irgendwelche sonstigen Umstände eine gewaltsame Veränderung des eigentlichen Körperbestandes annehmen lassen konnten. Diese sprunghaften Veränderungen der Dichte traf 
nicht allein Kastner, sondern auch Oppenheimer - letzterer bei etwas anderer Technik und bei (gesunden) Neugeborenen sogar in einem Zeitraum von 1 bis 2 mal 24 Stunden. Die Differenzen betrugen in seinen Fällen Stadler, Wiesner, Lautenschlag (Protokollnummern $10 / 11,26 / 27,36 / 37) 0,066,0,050,0,056$. Ein Index, der so jähen Schwankungen in gesunden Tagen ausgesetzt ist, kann nicht Ausdruck dessen sein, was man als Konstitution bezeichnet und die Einflüsse, die ihn so gewaltsam verändern, können nicht am Körpergewebsbestande angreifen.

Mehr oder weniger mit Gas erfüllte Körperräume sind insbesonders der Verdauungsschlauch und der Respirationsbaum; andere solche Räume haben eine zu geringe oder zu konstante Kapazität, um hier in Betracht zu kommen. Wir vermuteten in erster Linie in den Respirationsgasen mit ihrem stark wechselnden Volumen das störende Moment. Daß die Respiration ein Faktor ist, dem bei Volum- und Dichtebestimmungen des menschlichen Körpers Rechnung getragen werden müsse, wußten und berücksichtigten fast alle Untersucher. Man ging meist so vor, daß man bei dem Versuche von der Versuchsperson eine bestimmte Respirationsphase einhalten ließ. Dann konnte leicht nach Ermittlung der Atmungsgröße oder unter Annahme einer bestimmten mittleren Atmungsgröße bzw. Vitalkapazität auch das Körpervolumen und damit das spezifische Gewicht in anderen Phasen berechnet werden. Meeh - Vierordt geben die mittlere Körperdichte von sieben Männern im Alter von 16-45 Jahren bei stärkster Exspiration auf 1,0280, bei tiefster Inspiration auf 0,9670 (berechnet nach der Vitalkapazität) bzw. auf 0,9752 (direkt beobachtet) ${ }^{1}$ ) an; die Differenz, rund 0,06 , ist, wie man sieht, eine recht beträchtliche. Sie hängt $a b$ von der Größe der Vitalkapazität pro Kilogramm Körpergewicht; für diese berechnet sich bei Erwachsenen und älteren Kindern ca. $50 \mathrm{ccm}$; bei Säuglingen beträgt das Maximum der beobachteten Atmungsgröße pro Atemzug und pro Kilogramm Körpergewicht nur etwa 30, bei Neugeborenen $15 \mathrm{ccm}$ (Gregors und anderer Daten). Hiernach würde besagte Differenz in unserem Material immerhin noch 0,02-0,04 ausmachen (siehe hierüber auch Tabelle S. 420).

Die von Kastner benützte Technik, die Messung des t'berlaufwassers, schafft nun allerdings automatisch eine gewisse Niveaugleich-

1) Die Atmung bei unter Wasser getauchtem Rumpf erreicht nicht die maximale Inspiration. 
heit, indem hierbei naturgemäß das größte Volumen, jenes bei der tiefsten Inspiration, die während der Immersionsdauer eintritt, gemessen wird. Dadurch kann die beim Säugling natürlich nicht erreichbare Einhaltung einer bestimmten Atmungsphase einigermaßen ersetzt werden. Da aber das Verhalten der Kinder beim Versuch doch ein wechselndes war, nämlich nicht alle sich ruhig verhielten, einzelne schrien, bleibt eine gewisse Unebenmäßigkeit und damit ein Einwand bestehen. Nebst der Respirationsphase sind auch noch weitere Momente, wie Thoraxform, Obsoleszenz von Lungenteilen, Bronchialerweiterungen usw, auf die jeweilige Kapazität des Luftbaumes von Einfluß.

Jenem Einwand zu begegnen, den störenden Faktor der Respiration bei der Volumbestimmung gänzlich auszuschalten, waren wir verschiedentlich bedacht. Da eine streng gleichförmige Atmung und völlig proportionale Füllung der Lungenluftwege während des Untertauchens beim Säugling nicht garantiert werden kann, scheint uns die Wassertauchmethode heute für die vorliegenden Zwecke überhaupt ungeeignet. Man wird von ihr gänzlich absehen und ein prinzipiell anderes Verfahren wählen müssen. Als solches nahmen wir zunächst das folgende in Aussicht:

Das (bekleidete) Kind wird in einen Rezipienten gebracht, durch den ein atembares Gasgemenge (etwa aus 60\% Stickstoff, je $20 \%$ Wasserund Sauerstoff) strömt. Nachdem sich die Atmungswege damit gleichmäßig gefüllt haben, wird das Gasgemenge durch eingesaugte Luft nach einem Gasometer verdrängt, in dessen Inhalt dann der das Gasgemenge charakterisierende Bestandteil (hier der Wasserstoff) quantitativ ermittelt wird. Geht man gleich darauf mit dem Rezipienten ohne Kind ${ }^{1}$ ) in gleicher Weise vor, so erhält man eine Differenz zweier Wasserstoffbestimmungen, bzw. zweier Wasserwägungen, die ein direktes Mab für das gesuchte Körpervolumen des Kindes ohne die Hohlräume der Luftwege darstellt. Der störende Einfluß der Temperatur ist hier gering, und bei einer auf nur etwa $1 \mathrm{mg}$ genauen Wägung läßt sich noch die dritte Dezimale des spezifischen Gewichts ermitteln. Die Genauigkeit des Wägeverfahrens als solchen und die Verneunfachung des Gewichtes des Wasserstoffes bei seiner Oxydation kommen der Methode zustatten.

Eine solche Bestimmung des Körpervolumens ohne die lufthaltigen

I) Aber mit den Kleidern des Kindes. 
Hohlräume des Respirationstraktes würde ohne Zweifel einen Fortschritt bedeuten; sie durchzuführen würde sich aber nux dann lohnen, wenn damit die Aufgabe gelöst wäre, das Tolumen, also auch die Dichte des nicht gasförmigen Gesamtkörperbestandes beim Lebenden mit ausreichender Genauigkeit zu ermitteln. Dies dürfte aber nicht der Fall sein.

Bei einer Anzahl der Säuglinge mit auffallend geringer Körperdichte (nach Kastner) findet sich der Vermerk über einen stark gewölbten oder gar aufgetriebenen Unterleib in dem Krankenjournale, so z. B. in den Fällen Ehrenreich $(\mathrm{D}=0,8972)$, E. Fischer $(\mathrm{D}=0,9213)$, Immersberger $(\mathrm{D}=0,9379)$, Ad. Rauch $(\mathrm{D}=0,9187)$, Roß $(\mathrm{D}=0,9299)$ u. a. $\mathrm{m}$. Bei den drei erstgenannten wurde nach Rückgang der damit vielleicht zusammenhängenden Verdauungsstörung eine Erhöhung des spezifischen Gewichtes um 0,03-0,05 gefunden. Bei anderen Säuglingen blähte sich der Unterleib versus finem stärker auf und $\mathrm{K}$ ast ner konnte dann mitunter (Fälle Beer, Moraweck, Wesolek) das spezifische Gewicht wenige Tage vor und kurz nach dem Tode vergleichen. Hier war die Gesamtdichte nicht (wie der Exspirationszustand an der Leiche erwarten ließ) angestiegen, sondern gleichgeblieben oder sogar erheblich gesunken. Solche Beobachtungen legten den Gedanken nahe, daß wechselnde Gasfülle des Verdauungsschlauches die Gesamtkörperdichte mindestens ebenso stark beeinflusse wie der Lungenluftwechsel. Rechnung und Versuch bestätigten die Vermutung.

Bei Erwachsenen ist das Gesamtvolum der Gase in Magen und Darm sehr großen Schwankungen unterworfen und kann sich auf etliche Kubikdezimeter belaufen (Roith). Uber die Menge der Gase im Verdauungstrakt von Säuglingen konnte ich fast keine Daten in der Literatur finden - auch nicht bei Leo und bei Quest, die zahlreiche Analysen dieser Gase ausgefuhrt haben. Viel besprochen ist nur die vorwiegend forensisch bedeutsame Tatsache, da $\beta$ der Verdauungstrakt bei der Geburt vollständig gasfrei ist und sich bei atmenden Kindern unabhängig von der Nahrungsaufnahme vom Munde her allmählich mit Gas füllt (Breslau, 1865). In zwei Säuglingsleichen habe ich Gasmengen von $50 \mathrm{bzw} .100 \mathrm{ccm}$ in Magen und Darm angetroffen. Bei Verdauungsstörungen und intestinalen Zirkulationsstörungen mag sich aber nach klinischen und Obduktionsbefunden wohl oft ein Vielfaches dieser Menge unter den vorgewölbten Bauchdecken ansammeln. Jedenfalls konnten wir Säuglingen im 2.-5. Monat ohne Schwierigkeit - allerdings unter merklichem Ballonement - 150-250 ccm Luft 
per anum in den Dickdarm einbringen. In diesen Versuchen ergab sich folgendes:

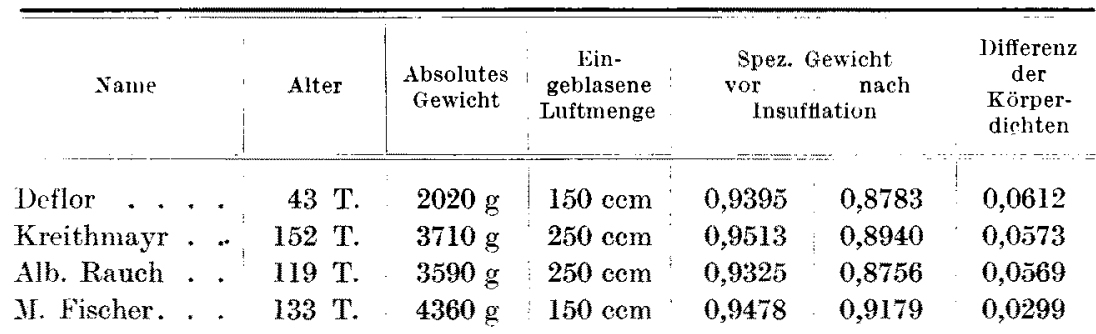

Die Beeinflussung der Gesamtkörperdichte durch Schwankungen im Gasgehalt des Verdauungsschlauches ist mithin eine so ausgiebige, daß es völlig nutzlos wäre, das spezifische Gewicht auf drei Dezimalen genau zu ermitteln, sofern man diesen Faktor nicht ausschließen oder beherrschen ${ }^{1}$ ) kann.

Um in übersichtlicher Weise zu veranschaulichen, welchen Einfluß auf die Gesamtkörperdichte - ermittelt nach dem Wassertauchverfahren - Schwankungen in der. Masse einzelner Körperbestandteile haben, wurde die Tabelle auf Seite 420 berechnet, die wohl geeignet ist aufzuklären, weshalb die Ausbeute an gesetzmäßigen Beziehungen dieser Größe zu anderen Faktoren eine so beschränkte geblieben ist.

Man erkennt, daß die sub 1 bis 16 in Betracht gezogenen, zum Teil sehr gewaltsamen Veränderungen des Körperbestandes an der Körperdichte zumeist weit weniger zum Ausdruck kommen, als die sub 17 bis 24 angenommenen Gasbestanddifferenzen, bezw. Bestimmungsfehler.

So können denn meines Erachtens Körperdichtenbestimmungen an Säuglingen beim gegenwärtigen Stande der Methodik den eingangs erwähnten Zwecken nicht dienen, und wir hätten von der Darlegung der dies erläuternden Befunde wohl gänzlich abgesehen, wenn nicht von anderer Seite in früherer Zeit und auch neuerdings wieder an das Verfahren Hoffnungen geknüpft worden wären.

1) Wengler deutet an, es wäre möglich, die jeweils im Magen-Darm vorhandene Gasfüllung bein Lebenden durch ihren EinfluB auf die Atmung zu ermitteln. Die einzige mir bekannt gewordene kurze Mitteilung des Autors (Deutsche med. Wochensehr., Vereinsbeilage 1902, S. 119) ermöglicht mir keine Beurteilung und Nachprüfung des Verfahrens. 


\section{'Tabelle,}

angebend die Veränderung, die das spezifische Gewicht des Gesamtkörpers nach Berechnung erleidet bei gewissen Veränderungen des Körperbestandes.

Angenommen ist als Ausgangsobjekt der Körper eines normalen, etwa 6 Wochen alten und $4000 \mathrm{~g}$ schweren Säuglings. Dieser Organismus habe cinen Gesamtbestand

an Panniculus adiposus von $400 \mathrm{~g}$ im spezif. Gewicht von 0,971 "quergestreifter Muskulatur , Wasser

,Salzen (Asche)

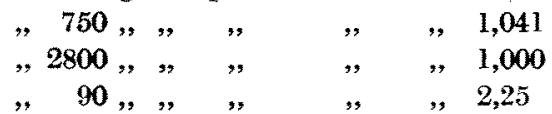

(Angaben nach Camerer, Vierordt u. a.)

Es verändert sich das spezifische Gewicht

1. Durch Terlust von $5 \%)(-0,0002+0,0003+0,0010$

2. "Zuwachs " $5 \%$ des Panniculus adi- $+0,0003-0,0002 \cdots 0,0007$

3. "Verlust "20\% posus um: $\left\{\begin{array}{c}-0,0013+0,0005+0,0030 \\ -0,0015\end{array}\right.$

4. "Zuwachs " $20 \%) \quad\left\{\begin{array}{l}+0,0015-0,0005 \\ +0,0027\end{array}\right.$

5. Durch Terlust ron $5 \%) \quad(-0,0011 \cdots 0,0004+0,0007$

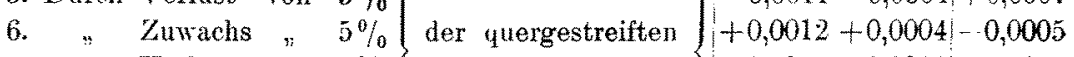

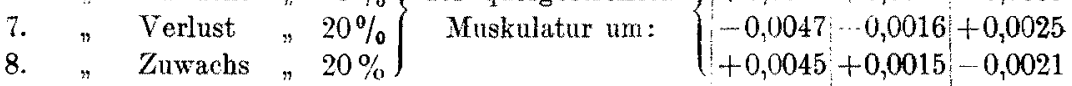

9. Durch Verlust von 5\%) $\quad(-0,0031+0,0000+0,0041$

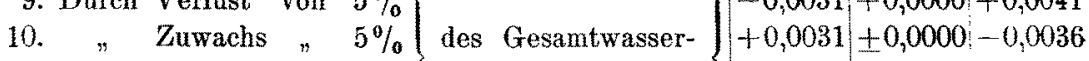

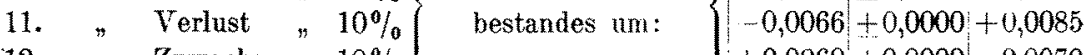

12. "Zuwachs $" 10 \%) \quad[+0,0060+0,0000-0,0070$

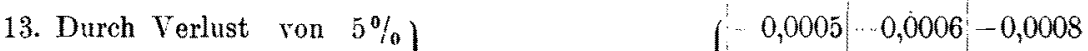

14. "Zuwachs " $5 \%$ des Gesamtaschen- $+0,0007+0,0006+0,0007$

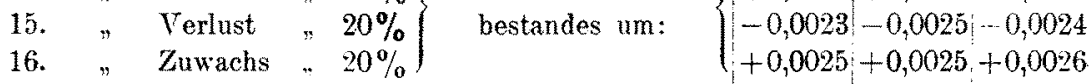

17. Durch Verminderung

18. , Vermehrung

19. "Verminderung

20. " Vermehrung

21. " Yerminderung

22. " Vermelurung

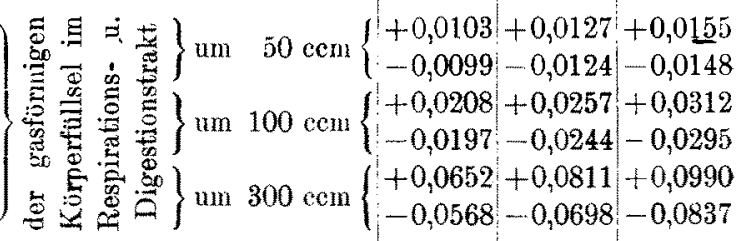

Durch Fehler bei der Tolumenbestimmung:

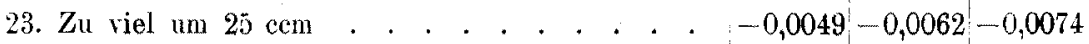

24. Zu wenig um $25 \mathrm{ccm}$. . . . . . . . . $+0,0052+0,0063+0,0077$ 
Die Erfüllung dieser Hoffnungen scheint uns aber doch nicht so völlig aussichtslos.

Dem Physiker dient zum Zwecke der Volumbestimmung von Körpern nebst der Tauchmethode auch die Methode der sogenannten Volumenometrie (Say, Leslie, Kopp, Régnault) ${ }^{1}$ ). Hierbei wird z. B. folgendermaßen vorgegangen: Man preßt in einen hermetisch abgeschlossenen leeren Rezipienten eine bestimmte Menge Luft. Dadurch entsteht eine gewisse Drucksteigerung. Diese Drucksteigerung vermehrt sich, wenn die Luftkapazität des Rezipienten vorher durch Einbringung einer Substanz (von fraglichem Volumen) verkleinert worden ist. Die Differenz der beiden Manometerstände liefert ein Ma 3 für das Volumen des eingeschlossenen Körpers. Es scheint nicht a priori ausgeschlossen, daß sich nach diesem Prinzip ein unseren Anforderungen genügendes Verfahren ausarbeiten lasse. Diese Forderungen sind folgende: die Methode muß das Körpervolumen des lebenden Säuglings auf ungefähr $10-20 \mathrm{ccm}$ genau zu ermitteln ermöglichen; sie muß handlich und schonend genug sein, um gleich der Wägung täglich angewandt werden zu können; sie muß das Volumen des Körpers abzüglich der gasgefüllten Hohlräume (Respirationsbaum und Verdau ngsschlauch) ergeben.

Ob letzteres beim Verfahren mit dem besagten Volumenometer der Fall sein werde, ist diskutabel - nicht so sehr hinsichtlich der Luft-, als der Verdauungswege. Es setzt entweder eine freie Kommunikation der betreffenden Hohlräume mit der Luft im Rezipienten voraus oder eine Nachgiebigkeit der Wandungen der Hohlräume. Ersteres trifft für die Luft-, letzteres in vermutlich ausreichendem Maße für die Verdauungswege zu; doch wäre dies erst durch besondere Versuche zu erweisen. Die Bedenken, die Donle gegen das von Gustav Jäger vor 33 Jahren zum ersten Male für solche Zwecke in Vorschlag gebrachte, aber noch niemals wirklich angewandte Verfahren äußert, dürften vielleicht weniger in Betracht kommen als die Fehlerquellen, die durch Temperatur- und Feuchtigkeitsunterschiede (Atmung) entstehen. Im Begriffe, den erforderlichen Apparat zu konstruieren und die Methode auszuarbeiten, behalten wir uns weitere Mitteilungen darüber vor.

Im folgenden soll noch dargelegt werden, welche Befunde sich bei fortlaufender Bestimmung des Körpergewichts und des Körpervolums

1) Zit. nach L. Pfaundler, Müller-Pouillets Lehrbuch der Physik usw. Braunschweig 1905. I. Bd., S. 463. 
von Säuglingen ergeben und welche Schlüsse daraus zu ziehen wären. Es sei gleich bemerkt, daß diese Schlüsse trotz den oben gemachten Einwendungen gegen das Tauchverfahren Geltung haben. Die Frage ist zunächst prinzipiell zu beantworten; darauf sollen einige konkrete Beispiele aus Kastners Beobachtungsreihen erläutert werden.

¿́berlegt man, welche Möglichkeiten sich bei der Veränderung der drei Größen (Körpervolumen, absolutes und spezifisches Gewicht) allgemein ergeben, so gelangt man zu folgendem Schlusse: Das absolute Gewicht kamn sich vermehren, kann erhalten bleiben oder kann sich vermindern; in jedem dieser drei Fälle kann das Volumen zunehmen, gleichbleiben oder abnehmen. Bei sieben von diesen neun Eventualitäten ist das Verhalten des spezifischen Gewichtes eo ipso gegeben. In den zwei übrigbleibenden Fällen aber kann die Körperdichte ansteigen, gleichbleiben oder sinken, je nachdem die Veränderung des absoluten Gewichtes oder jene des Volumens überwiegt, oder aber beide ebenmäßig sind. Man erhält also dreizehn mögliche Spezialfälle nach folgendem Schema (Fig. 1).

Es entsteht nun die Frage, welche Schlüsse, aus der Veränderung der drei Größen in einem bestimmten Zeitraume hinsichtlich der Qualität der angesetzten, ersetzten oder geschwundenen Masse gezogen werden können. Da ergibt denn die einfache Ĺberlegung folgendes:

Fall Ala. Der Körper hat angesetzt eine Masse' ${ }^{1}$, die spezifisch schwerer ist, als der Gesamtkörper war.

Fall A1b. Die angesetzte Masse hat dieselbe Dichte wie der Gesamtkörper.

Fall A lc. Die angesetzte Masse hat eine geringere Dichte als der Gesamtkörper.

Fall B1. Die angesetzte Masse ist Gas ${ }^{2}$.

Fall A2. Die Ersatzmasse ist spezifisch schwerer, als der Gesamtkörper war.

Fall B2. Die Ersatzmasse ist spezifisch gleichschwer, wie der Gesamtkörper war.

Fall C2. Die Ersatzmasse ist spezifisch leichter, als der Gesamtkörper war.

Fall B3. Die verlorene Masse war Gas.

1) bzw. Massen, deren Durchschnitt spezifisch sohwerer ist usw.

2) Recte eine Masse, deren spezifisches Gewicht jenen der Luft innerhalb der Fehlergrenzen der Bestimmung gleichkommt. In Betracht kommt nur Lungenluft und Magendarmgas. 
Fall C3a. Die verlorene Masse hatte eine geringere Dichte als der Gesamtkörper.

Fall C3b. Die verlorene Masse hatte dieselbe Dichte wie der Gesamtkörper.

Fall C3c. Die verlorene Masse hatte eine größere Dichte als der Gesamtkörper.

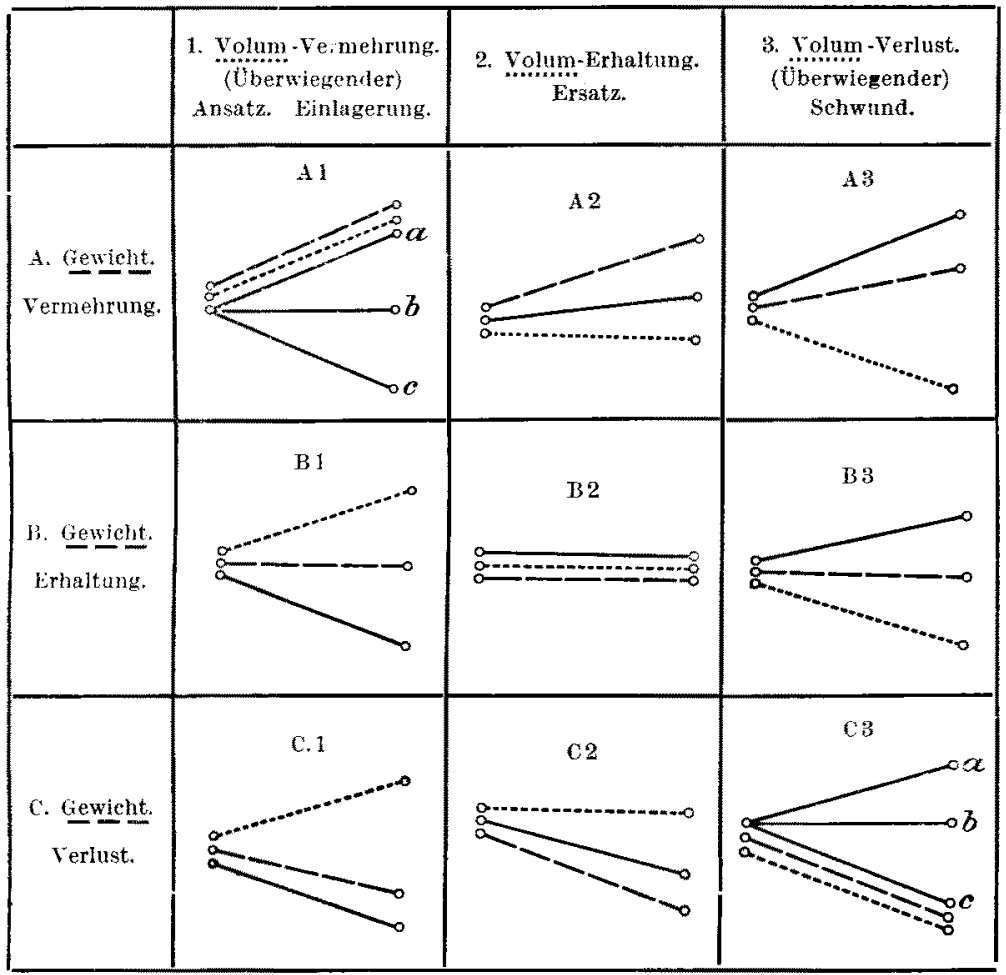

Fig. 1.

Die - - Linie zeigt das Verhalten (Ansteigen, Konstanz, Abfallen) des absoluten Körpergewichtes, die -.........-.-. Linie jenes des Körpervolumens, die jene des spezifischen Körpergewichts an.

Etwas kompliziertere Verhältnisse müssen in den Fällen A 3 und C 1 vorgelegen haben. Bei A3 muB nebst einer spezifisch leichteren Masse überdies auch Gas verloren gegangen sein, im Fall C1 muß Gas angesetzt worden und eine spezifisch schwerere Masse verloren gegangen sein.

Die meisten der theoretisch möglichen Fälle sind in Kastners Material tatsächlich vertreten. Zur Erläuterung dienen die nach 
Kastners Bestimmungen angefertigten Kurventabellen auf beiliegender Tafel IV.

Erstes Beis piel: Entwicklung eines gesunden, bis zur 10. Lebenswoche an der Mutterbrust ernährten, dann allmählich auf Kuhmilchmischung abgestillten Kindes (Fall Brand, Fig. 1 der Tafel).

Man sieht hier die Typen Ala, Alb, Ale und A2 nach obiger Bezeichnungsweise abwechselnd vertreten oder, mit anderen Worten, bei andauernder Zunahme des Körpergewichtes ist das Körpervolumen meist gleichfalls angestiegen (in den Perioden „A $1^{\text {": }} \mathrm{I}$, II, IV bis VI), und zwar teils weniger steil als das Körpergewicht (in den Perioden „A 1 a“: I, V); teils ungefähr gleich steil wie das Gewicht (in der Periode „A $1 b^{\text {" }}$ : IV); teils steiler als das Körpergewicht (in den Perioden "Al c": II, VI); gelegentlich aber ist es - innerhalb der Fehler der Bestimmung - konstant geblieben (in den Perioden „A 2 “: III und VII).

Zwischen dem 46. und 56., dem 60. und 77. Lebenstage erfolgte ein überwiegender Ansatz, eine Vermehrung der (durch die Tauchmethode bestimmten Gesamt-) Körpermasse. Dabei war die angesetzte Masse im Durchschnitt teils dichter, teils gleich dicht, teils minder dicht als der Gesamtkörper.

Zwischen dem 56. und 60., dem 77. und 111. Lebenstage abar (also während der Abstillungsdauer), während der die Körpergewichtskurve gleichmäßig weiter anstieg, fand eine solche Vermehrung nicht mehr statt, sondern bei gleichbleibendem Gesamtvolumen nur eine innere Umlagerung oder Auswechslung, deren Endeffekt der Ersatz einer Masse von geringerer durch eine solche von größerer Dichte war: Bøi bloßer Betrachtung der Körpergewichtskurve und der übrigen gewöhnlich registrierten Erscheinungen kommt eine Verschiedenheit dieser aufeinanderfolgenden Entwicklungsperioden übərhaupt gar nicht zum Ausdruck.

Diese aus dem Verhalten der Gewichts- und Volumkurven gezogenen Schlüsse sind zwar formell unbedingt korrekt und unangreifbar; ihre Tragweite für die Zwecke klinischer Analyse wird aber doch wesentlich dadurch beeinträchtigt, da $\beta$ die Veränderungen im Bestande des Bronchial-, des Blasen- und des Magendarminhaltes einbezogen sind. So wäre es z. B. möglich, daß der Wechsel, der nach dem 50. Lebenstage eintrat (Umschwung von Ala in Alc) durch eine bloße Vermehrung der Darmgase bedingt gewesen sei; diese Vermehrung hätte dann freilich - wie die Rэchnung ergibt - etwa $180 \mathrm{ccm}$ betragen 
müssen. Von einer stärkeren Aufblähung der Därme oder von Koliken war aber während der ganzen Beobachtungsdauer nichts zu bemerken. Es wäre zu erforschen, welche Bestandveränderungen an Darmgasen die Grenze klinischer Wahrnehmbarkeit erreichen.

Nach den von Kastner mitgeteilten Durchschnittswerten des spezifischen Gewichtes bei älteren und jüngeren Säuglingen wäre der Wachstumstypus A 1c: Vermehrung von Gewicht und Volumen bei überwiegender Zunahme des letzteren, also bei Verminderung des spezifischen Gewichtes, das ist der Ansatz einer im Durchschnitt leichteren Masse der für längere Periode des Säuglingsalters und wohl darüber hinaus im ganzen physiologische.

Zweites Beispiel. Langdauernde Unterernährung eines Kindes infolge schweren, habituellen Erbrechens (Fall Ries, Fig. 2 der Tabelle).

Perioden leidlicher Zunahme vom Typus A 1a und A 1c wechseln hier mit Perioden, in denen Gewicht und Volumen abnehmen; dabei überwiegt teils der Gewichtsverlust (C3c in Periode II), teils der Volumverlust (C3a in Periode VI). In der IV. und V. Periode macht sich ein ziemlich steiler Anstieg von Gewicht und Volumen bemerkbar. Hier stand das Kind unter dem Einfluß einer Arsenmedikation. Diese scheint den Ansatz einer im Durchschnitt spezifisch leichteren Masse bewirkt zu haben (A 1c), welcher Ansatz allerdings noch im weiteren Verlauf der Kur wieder vollständig zurückging. Im letzten Monate der Beobachtung hatte sich der Zustand des Kindes wesentlich gebessert.

Drittes Beispiel. Langsame Reparation eines Zustandes von langdauernder Wachstumsstörung, vermutlich endogenen Ursprunges bei einem Säugling, der bei der Aufnahme das Bild der Atrophie bot. (Vorgeschichte fehlt, Tuberkulinproben negativ. Fall Buff, Fig. 3 der Tafel.)

Das Kurvenbild weicht bemerkenswerterweise wenig von jenem im ersten Beispiel ab. In der IV. Periode trat (unter leicht dyspeptischen Erscheinungen) nach vorausgegangener steiler Zunahme ein Rückschlag ein, den Schloss wohl als „,Reversion“ bezeichnen würde. Der labile Ansatz müßte hier nach dem Verhalten der Körperdichte aus spezifisch leichteren Massen bestanden haben, deren Zusammenbruch andere mit sich riß. Für die Analyse späterer ähnlicher Vorkommnisse und des ,Etappenwachstums“ in der V. Periode reicht leider die Anzahl der Volumbestimmungen nicht aus.

Viertes Beispiel. Bronchialdrüsentuberkulose bei einem recht gut gedeihenden Kind. (Fall Wieser, Tabelle Fig. 4.) 
Die Kurve zeigt, wie sich bei unserem Vorgehen die Bildung und Stauung größerer Gasmassen im Darm bei Meteorismus darstellen. Dieser Zustand trat in dem Falle beim Rüekgang von Zwiemilchernährung auf Magermilch mit Nährzucker in der Periode II plötzlich ein. Man sieht, wie sich bei kaum verändertem Gewichtsanstieg Volumund Dichtekurven schneiden und eine gewaltsame Veränderung der Situation hervorrufen. Es handelt sich hier allerdings um den bei weitem ausgesprochensten Fall unserer Beobachtung. Unter Anstieg von Gewicht und Volumen tritt später ein teilweiser Ausgleich ein. In der Periode IV wurden die Gewichts- und die Volumzunahme vorübergehend durch eine katarrhalische Affektion unterbrochen.

Fünftes Beispiel. Chronische Entwicklungsstörung unter dem Bilde der Atrophie, schwerer Anlagefehler (wohl Erbschaden, weil familiäre Polymortalität). Keine Tuberkulin-Anaphylaxie. (Fall Avenius, Fig. 5 der Tafel).

Die Kurventabelle zeigt die merkwürdige Reaktionslosigkeit, die hier durch fast 2 Monate bestand. (Die Gewichtskurve bildete auch im Original während der Periode III nur einen flachen Sattel.) Verhalten von Körpervolumen und -dichte machen es unwahrscheinlich, daß sich während dieser Zeit eine innere Umsetzung ereignet und eine latente Reparation angebahnt hätte. In der Tat hat das Kind erst viel später zuzunehmen begonnen. Aus dieser Zeit fehlen Volumbestimmungen.

Diese Beispiele sollen lediglich erläutern, in welcher Weise Volumund Dichtebestimmungen bei lebenden Säuglingen verwertet werden könnten, wenn es gelänge, eine den oben aufgestellten Forderungen genügende Methodik zu finden und fortlaufend anzuwenden.

\section{Literaturverzeichnis.}

Gregor, Untersuchungen über die Atmungsgröße des Kindes. Archiv f. Anat. u. Physiol. 1902.

Kastner, Körpervolumen und spezifisches Gewicht von Säuglingen. Diese Zeitschrift. Vorhergehende Arbeit.

Oppenheimer, Über eine Methode zur Bestimmung des Volumens bei Säug* lingen. Diese Zeitschr. Band III. Heft 3.

Meeh, Volummessungen des mensehlichen Körpers usw. Zeitsehr. f. Biol., N. F. 13. 1894.

Jäger, Seuchenfestigkeit und Konstitutionskraft usw. Leipzig 1878.

Schloss, Die Pathologie des Wachstums im Säuglingsalter usw. Berlin 1911. Roith, Die Füllungsverhältnisse des Dickdarms. Anat. Hefte 20. 1903. 
Körpervolum- und Körperdichtebestimmung am lebenden Säugling.

Leo, Über den gasförmigen Mageninhalt bei Kindern im Säuglingsalter. Zeitschr. f. klin. Med. 41. 1900.

Quest, Untersuchungen über Darmgase bei Säuglingen mit Tympanitis. Jahrb. f. Kinderheilk., N. F. 59.

Breslau, Über Entstehung und Bedeutung der Darmgase beim neugeborenen Kinde. Monatsschr. f. Geburtshilfe 1865.

Vierordt, Anatomische, physiologische und physikalische Tabellen. Jena 1906. Krause, Anatomie, Bd. II (zit. nach Vierordt). 


\section{after Tafel 3}
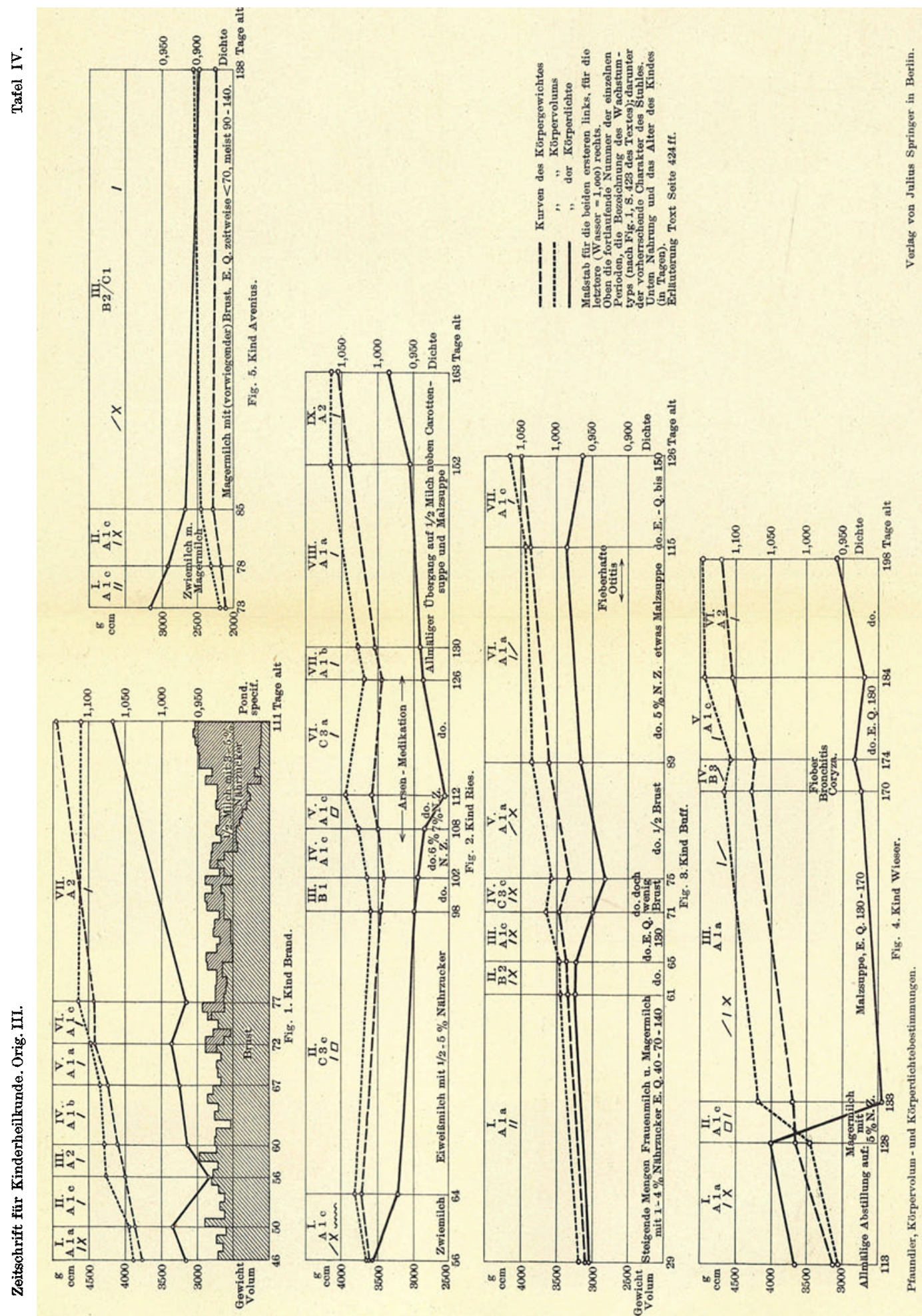